\title{
Technè
}

La science au service de l'histoire de l'art et de la préservation des biens culturels

$44 \mid 2016$

Archives de l'humanité : les restes humains patrimonialisés

\section{Ultimes soins pour des crânes surmodelés du Vanuatu}

Ultimate care bestowed upon carved skulls from Vanuatu

\section{Régis Prévot}

\section{(2) OpenEdition}

12 Journals

Édition électronique

URL : http://journals.openedition.org/techne/1196

DOI : 10.4000/techne. 1196

ISSN : 2534-5168

Éditeur

C2RMF

Édition imprimée

Date de publication : 1 novembre 2016

Pagination : 118-121

ISBN : 978-2-7118-6339-6

ISSN : 1254-7867

Référence électronique

Régis Prévot, "Ultimes soins pour des crânes surmodelés du Vanuatu », Technè [En ligne], 44 | 2016, mis en ligne le 19 décembre 2019, consulté le 22 juillet 2020. URL : http://journals.openedition.org/ techne/1196; DOI : https://doi.org/10.4000/techne.1196

\section{(c) (i) (9)}

La revue Technè. La science au service de l'histoire de l'art et de la préservation des biens culturels est mise à disposition selon les termes de la Licence Creative Commons Attribution - Pas d'Utilisation

Commerciale - Pas de Modification 4.0 International. 


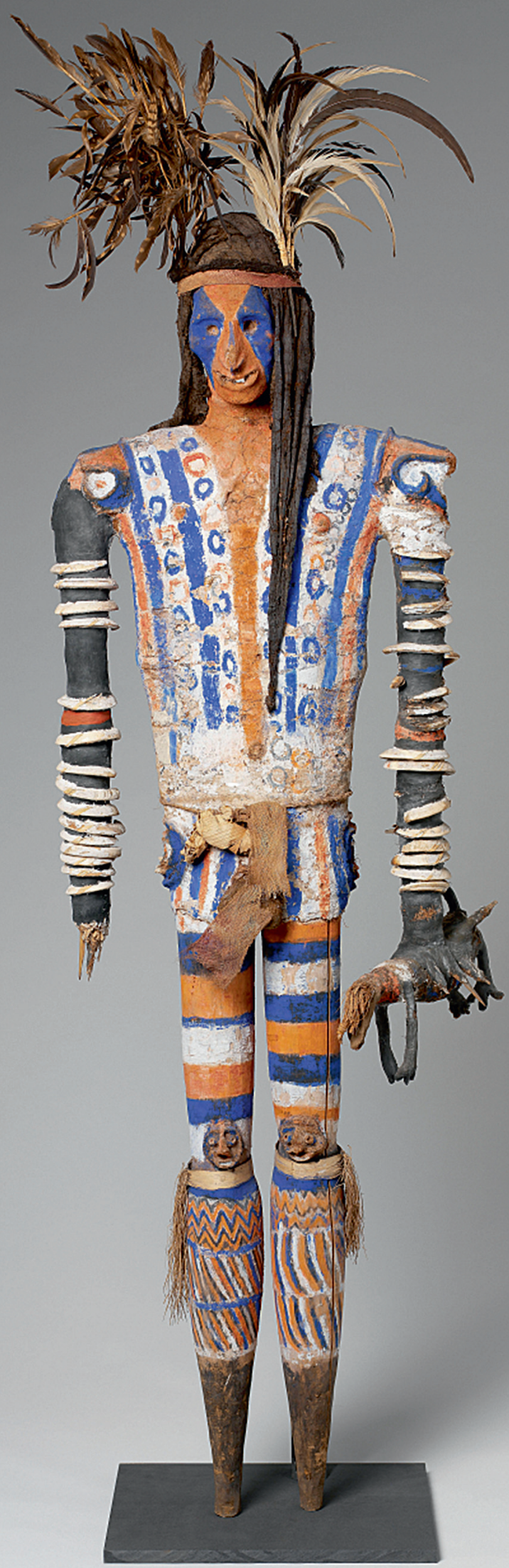

Fig. 1. Mannequin funéraire avec son crâne surmodelé -

Rambaramb -, île de Malékula au Vanuatu, musée du quai Branly, $\mathrm{n}^{\circ}$ inv. 72.1931.1.4.

(c) Musée du quai Branly - Jacques Chirac, Dist. RMN-Grand Palais/ P. Gries/B. Descoings. 


\section{Régis Prévot Ultimes soins pour des crânes surmodelés du Vanuatu}

Ultimate care bestowed upon carved skulls from Vanuatu

Résumé. Après le rappel de quelques notions sur la tradition des crânes surmodelés du Vanuatu, l'auteur s'attache à analyser l'état de quatre crânes ayant séjourné durant plusieurs décennies dans un musée français, avant d'aborder le contexte et les modalités de leur restauration jusqu'à leur mise en réserve.

Mots-clés. Restes humains, crâne surmodelé, Vanuatu, enduit végétal, restauration, nettoyage par compresses.
Abstract. After recalling some notions about the tradition of carving skulls in Vanuatu, the author of this paper analyses the condition of four skulls that had been held in a French museum for several decades. He then explains the context and modalities of their restoration, before they were placed in storage.

Keywords. Human remains, carved skull, Vanuatu, plantbased coating, restoration, cleaning with compresses.
En 1993, le Musée départemental breton de Quimper nous a sollicité ${ }^{1}$ pour assurer le traitement de quatre crânes surmodelés du Vanuatu en vue de leur exposition. Ils font partie d'un lot de huit crânes probablement entré dans les collections du musée à la fin du XIX ${ }^{\mathrm{e}}$ siècle ou au tout début du XX⿳一巛工 ${ }^{\mathrm{e}}$ siècle ${ }^{2}$. Lors d'expéditions d'exploration dans les mers du Sud, il est très courant que de tels objets aient été rapportés par un marin $^{3}$ et se soient ensuite retrouvés dans un musée sans plus d'information sur leur collecte et leur entrée dans les collections.

Il s'agit ici de crânes de natifs de l'archipel du Vanuatu (ex-Nouvelles-Hébrides), archipel qui se situe dans la région mélanésienne du Pacifique sud, à l'est de l'Australie et de la Nouvelle-Guinée et au sud des Îles Salomon. Il est constitué d'un chapelet d'îles dont la plus importante est l'île de Malékula d'où proviennent ces crânes surmodelés à l'occasion d'une coutume uniquement pratiquée dans le sud de l'île.

\section{Tradition autour des crânes surmodelés du Vanuatu}

"Les crânes sont surmodelés ${ }^{4}$ avec un mélange de matières végétales, de sève d'arbre à pain et d'autres jus extraits de plantes, on reproduisait alors, le plus fidèlement possible, les traits du visage du défunt. Le crâne était alors fixé sur un mannequin funéraire de taille humaine appelé Rambaramb (fig. 1). Cette effigie servait durant les différentes cérémonies mortuaires, puis le mannequin était abandonné ${ }^{5}$ et le crâne surmodelé déposé dans la Maison des hommes ${ }^{6}$ ", coutume pratiquée dans le sud de l'île de Malékula 7 .

Après obstruction des orifices avec des feuilles de végétaux et surmodelage à l'enduit ${ }^{8}$, les faces des crânes étaient peintes alors que les cheveux et le cuir chevelu pouvaient soit être préservés, soit être remplacés par une toile d'araignée formant chevelure. Pour deux des crânes, la calotte crânienne est à nu. Les couleurs que l'on retrouve sur les différentes faces sont le noir, le noir/blanc, le noir/blanc/rouge et le noir/ blanc/ocre rouge/ocre jaune. Si le surmodelage visait à individualiser le crâne de manière réaliste et au moyen de signes distinctifs, des marques significatives d'appartenance à des grades atteints par le défunt étaient aussi présentes ${ }^{9}$.

\section{État des crânes avant intervention}

De façon générale, les altérations sont similaires sur chacun des crânes. Outre un empoussièrement particulièrement important, les salissures sont incrustées dans l'enduit et dans la couche picturale (fig. 2). Les cheveux et le cuir chevelu disparaissent sous les salissures. Ils abritent des brindilles et quelques cocons d'insectes. L'aspect d'origine des surfaces peintes n'est plus discernable.

L'enduit de surmodelage est très fissuré, craquelé. Il est parfois lacunaire au niveau du menton, de la tempe. Il présente des écailles, des soulèvements. Il est, tout comme la couche picturale, érodé en différents endroits - oreilles, arcades sourcilières, pommettes des joues.

Régis Prévot, ingénieur d'études, en charge du domaine de l'ethnographie extra-européenne, département Restauration et département Conservation préventive, C2RMF (regis.prevot@culture.gouv.fr). 


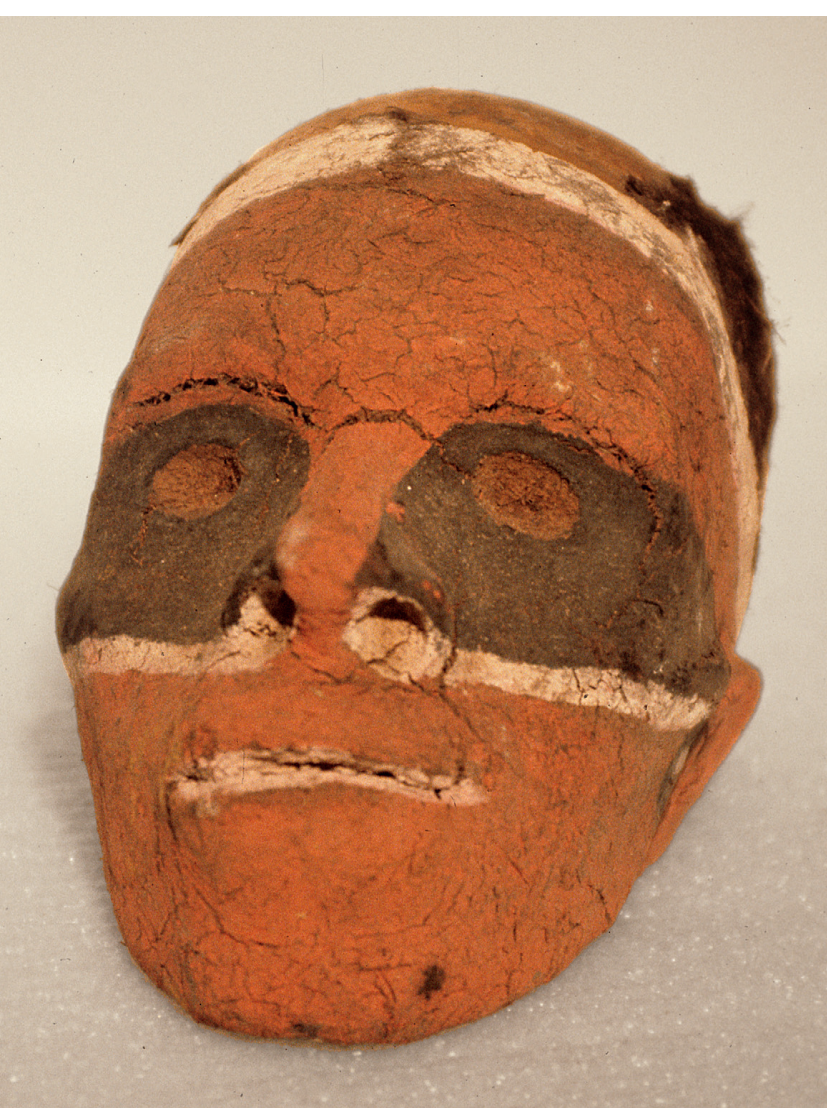

Fig. 2. Encrassement très important du crâne surmodelé, peint en rouge, noir et blanc. Musée départemental breton de Quimper. (C) R. Prévot.

Pour l'ensemble de ces modelages, deux oreilles sont manquantes alors que deux autres sont simplement désolidarisées, dont l'une avec l'enduit dissocié en plusieurs éclats.

Les cuirs chevelus en partie lacunaires, sont partiellement désolidarisés de la calotte crânienne. Les cheveux sont très cassants, fragilisés et peu adhérents aux cuirs chevelus.

\section{Interventions de restauration}

L'objectif de cette intervention était de stabiliser l'état des crânes et de leur redonner une lisibilité satisfaisante.

Elle a d'abord débuté par un dépoussiérage réalisé à la brosse douce pour pousser la forte couche de salissures mobiles vers la buse du microaspirateur. Son efficacité a permis de redonner un certain lustre aux cheveux et a dû être complétée par un nettoyage au coton-tige humecté d'eau déminéralisée pour les calottes crâniennes à nu.

À la suite de tests sur les surfaces peintes, la capacité migratoire de l'eau pour véhiculer les salissures incrustées dans la matière picturale a été utilisée. La surface a ainsi été légèrement humidifiée par application généralisée de compresses de ouate de cellulose imprégnées d'eau déminéralisée et recouvertes d'un film de Mylar afin de forcer la migration vers l'objet. Après un temps très court, 10 à 20 minutes, les compresses étaient remplacées par des compresses sèches laissées à l'air libre (sans Mylar) pour, à l'inverse, forcer la migration de l'eau, de l'objet vers la compresse. Très rapidement, l'encrassement de la compresse, mais aussi, après séchage, l'état de surface de l'objet traduisaient l'efficacité du traitement. Il a parfois été nécessaire de renouveler l'application deux à trois fois maximum pour obtenir un résultat satisfaisant et homogène.

Les écailles, les soulèvements d'enduit, les oreilles décollées, tout comme le cuir chevelu désolidarisé ont été consolidés, refixés au Paraloïd B72 dans l'acétone. L'adhésion des cheveux au cuir chevelu a été renforcée par une pulvérisation de Klucel G à $2 \%$ dans l'acétone. Elle a en outre contribué au nettoyage des cheveux et à une reprise partielle de leur souplesse.

La volonté de respecter au maximum l'intégrité de ces restes humains nous a amené à faire le choix d'une intervention minimaliste sur ces objets. Aucun élément ne fournissait d'orientation quant au modelé à donner aux oreilles manquantes, d'autant moins qu'aucune différence notable de modelé entre les deux oreilles n'était constaté pour les crânes intègres. La lisibilité satisfaisante des crânes avec une oreille manquante a motivé la décision de ne pas réintégrer les oreilles lacunaires (fig. 3). Une interprétation réaliste de ces crânes est tout à fait possible pour le public à partir du profil intact.

\section{Préservation sur le long terme}

À l'issue de ces soins, il a été décidé de réaliser, pour chaque crâne, un réceptacle individuel en carton non acide, afin de leur assurer tranquillité, protection et préservation en réserve

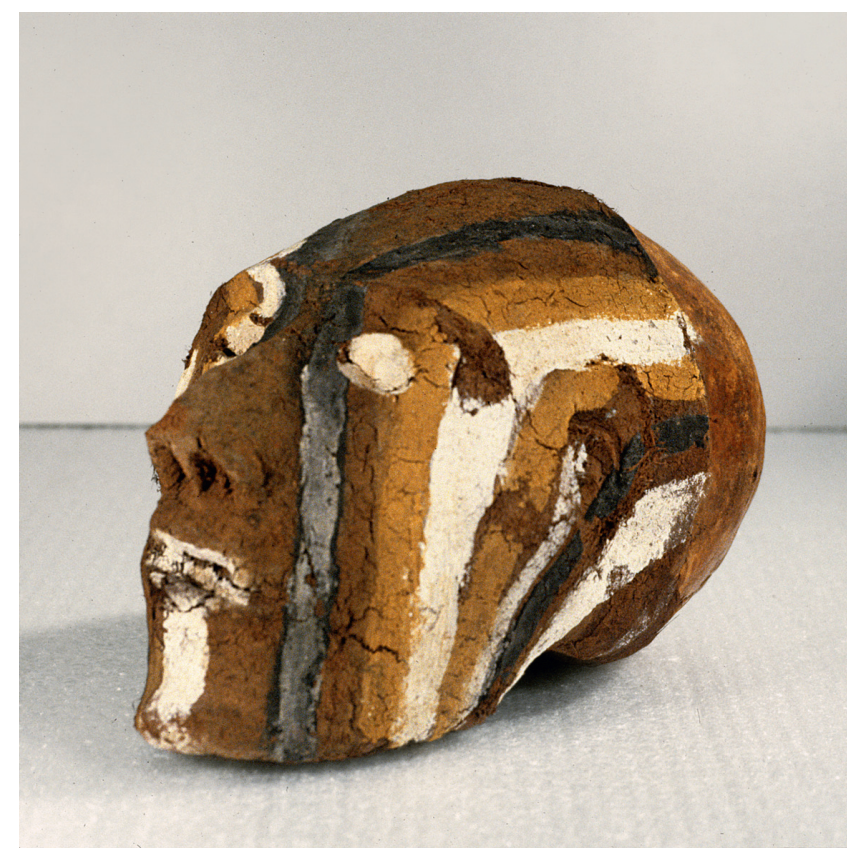

Fig. 3. L'oreille du crâne toujours lacunaire après traitement. Musée départemental breton de Quimper. (C) R. Prévot. 
(fig. 4). Ces supports de conditionnement facilitent les déplacements et les transports lors de prêts pour exposition. De même, ils rendent plus aisées les manipulations dans le musée, notamment pour leur présentation durant les Journées du patrimoine, seul moment de présentation au public pour ce musée dont les thématiques scientifiques ne recouvrent pas les collections ethnographiques.

Au cours de ces traitements ou d'interventions similaires sur des restes humains, il nous arrive très souvent d'avoir, de la part de collègues et d'observateurs extérieurs, des questions, des remarques sur notre état d'esprit face à ce type d'objet. Ces interlocuteurs se mettant en effet à notre place et exprimant une réelle réserve, une forte appréhension, presque une peur quant au fait d'intervenir sur des restes humains. Nous avouons ne pas partager leur ressenti. Le fait que la patrimonialisation et la muséification des restes humains soient sujets à controverse ne peut justifier qu'on les ignore. Bien au contraire, encore moins que tout autre objet, parce qu'ils sont des restes humains, ils ne doivent en aucun cas être oubliés, abandonnés au sein des collections. Les soins pratiqués doivent l'être dans le respect des traditions culturelles et des croyances de leur groupe ethnique d'origine. Par ces soins, une certaine dignité physique peut être redonnée à ces dépouilles. C'est une façon d'accorder au défunt le respect minimum qui lui est dû, mais c'est aussi reconnaître ces peuples, ces groupes ethniques, leurs traditions culturelles, leur histoire. C'est dans cet état d'esprit que ces soins leur ont été prodigués.

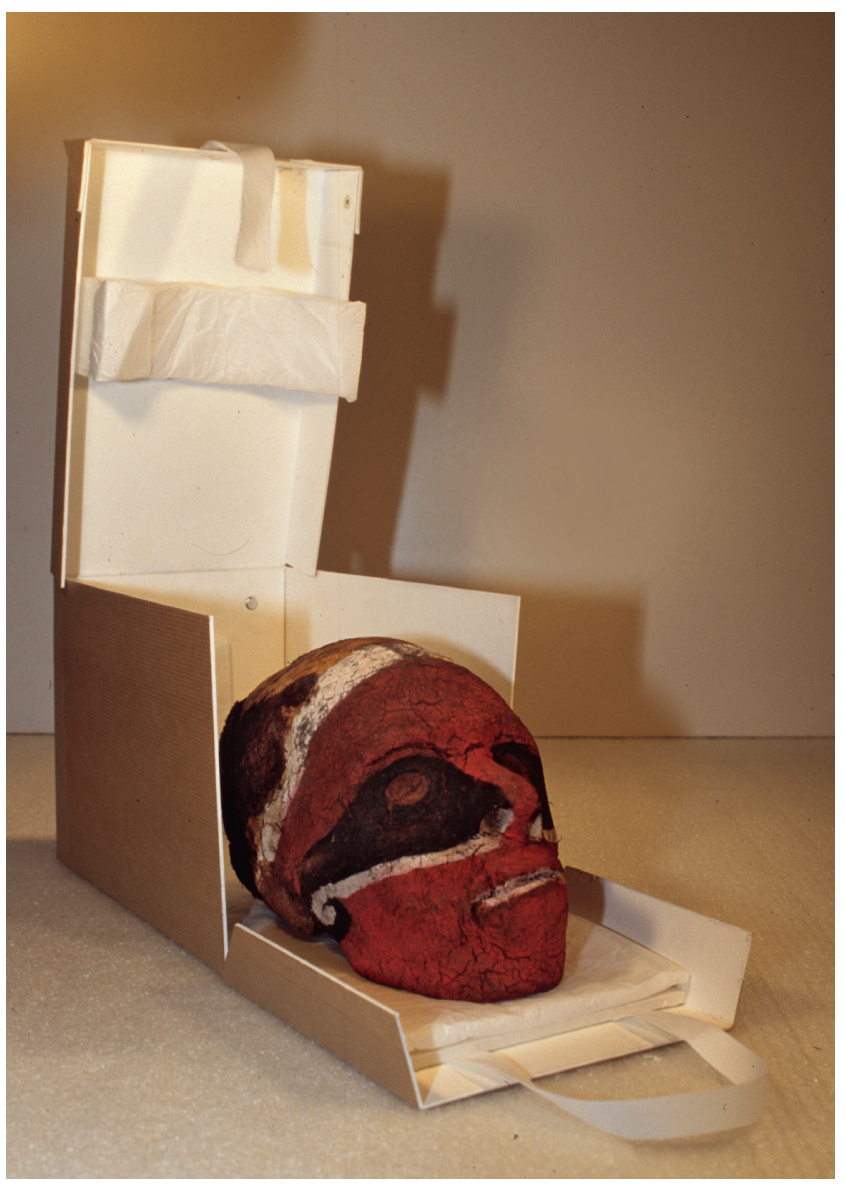

Fig. 4. Après restauration : transfert d'un des crânes dans son réceptacle de mise en réserve. Musée départemental breton de Quimper. () R. Prévot.
Notes

1. À cette date, l'auteur était alors restaurateur au musée des Arts d'Afrique et d'Océanie, spécialisé dans le traitement des collections ethnographiques.

2. Le musée ne dispose d'aucune information sur la date et le contexte d'entrée des crânes dans ses collections.

3. De nombreux objets conservés dans les musées français ont été rapportés par des marins lors de voyage dans les mers du Sud.

4. J. P Meyer A., 1995, p. 424.

5. «Dans la forêt où elle se décomposait sans faire l'objet de soins particuliers. ", in La mort n'en saura rien, p. 230.

6. Pour le Vanuatu, la pratique du surmodelage de crâne semble s'être limitée au sud de l'île de Malékula.

Voir La mort n'en saura rien, p. 166.

7. Ces effigies « étaient réservées aux défunts initiés aux rituels nalawan, newinbür et nimanggi. Des grades acquis au sein de ces sociétés dépendaient le nombre et les différentes parures que l'on disposait sur le mannequin », in La mort n'en saura rien, p. 230.
8. L'enduit ou «pâte végétale était un mélange de sciure de liane râpée, de moelle de fougère, de lait de noix de coco et de pâte de fruit à pain (Deacon, 1934, p. 545 ;

Guiart, 1965, p. 21) ». La mort n'en saura rien, p. 166.

9. La mort n'en saura rien, "Visages reliquaires », p. 118.

\section{Bibliographie}

Cadot L., 2009, «En chair et en os : le cadavre au musée. Valeurs, statuts et enjeux de la conservation des dépouilles humaines patrimonialisées ", École du Louvre/ RMN, Paris.

Delaporte F., 2014, « Les rapports de l'homme avec les restes humains aujourd'hui ", Support/Tracé14, p. $79-84$.

Guillemard D., 2010, «En 2007, sous le titre "Conserver les restes humains patrimonialisés. Enjeux éthiques et approche pratique”, Laure Cadot présentait son mémoire de master de conservation-restauration ", Conservation-Restauration des Biens Culturels 28, p 62-63.

J.-P. Meyer A., Art océanien, Könemann, Cologne, 1995.

Quétel E., «Conserver et restaurer les restes humains : exemple d'une étude réalisée sur cinq peaux humaines tatouées, conservées au musée Testut Latarjet de Lyon ", Support/Tracé 14, p. 85- 91.

Exp. Port-Vila, Nouméa, Bâle, Paris, de 1996 à 1998, Vanuatu Océanie, Arts des îles de cendre et de soleil, [Port-Vila, Nouméa, Bâle, Paris, de 1996 à 1998,], Réunion des musées nationaux.

Exp. Paris, 1999 : "La mort n'en saura rien ", Reliques d'Europe et d'Océanie, [Exposition. Paris, musée des Arts d'Afrique et d'Océanie, Paris, 1999], Réunion des musées nationaux. 